\title{
Report on fundamental modeling of irradiation-induced swelling and creep in FeCrAl alloys
}

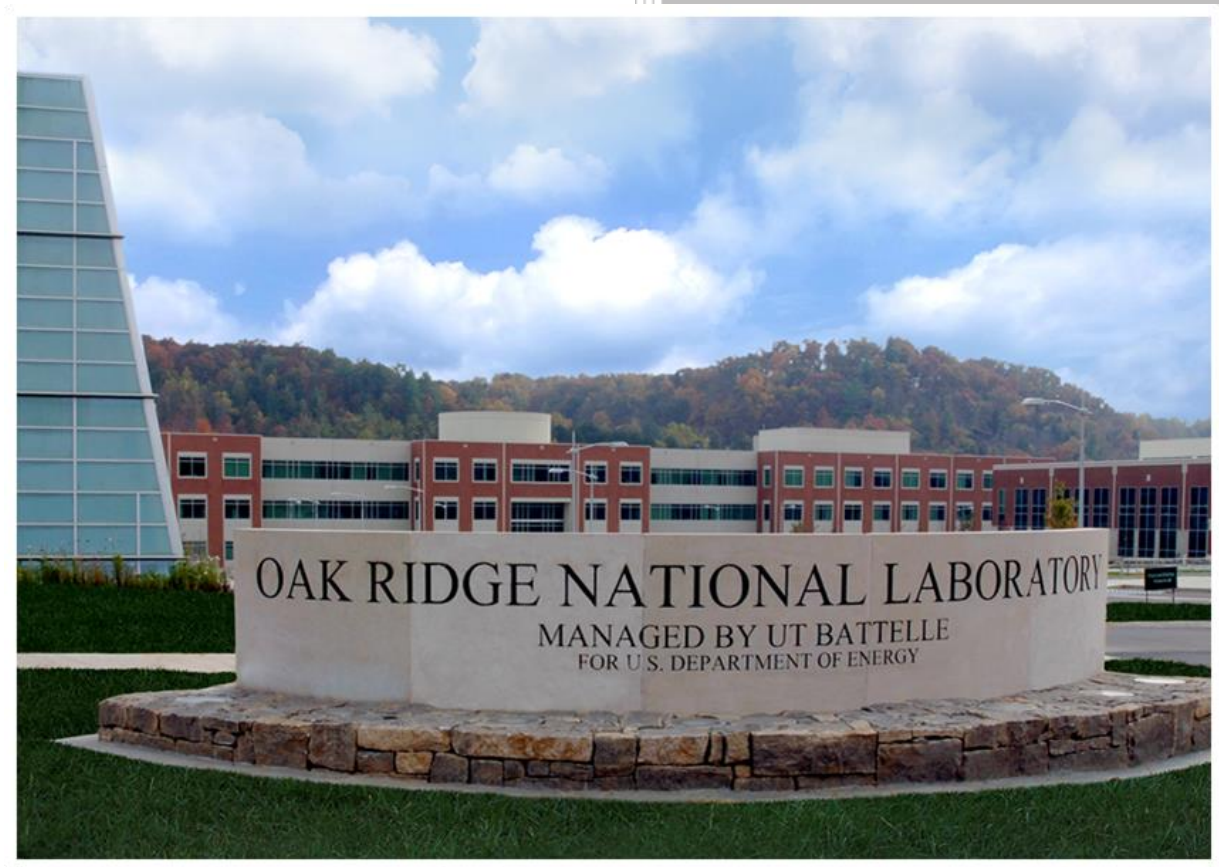

Aaron Kohnert Dwaipayan Dasgupta Brian Wirth

September 23, 2016

Approved for public release. Distribution is unlimited. 


\title{
DOCUMENT AVAILABILITY
}

Reports produced after January 1, 1996, are generally available free via US Department of Energy (DOE) SciTech Connect.

Website http://www.osti.gov/scitech/

Reports produced before January 1, 1996, may be purchased by members of the public from the following source:

\author{
National Technical Information Service \\ 5285 Port Royal Road \\ Springfield, VA 22161 \\ Telephone 703-605-6000 (1-800-553-6847) \\ TDD 703-487-4639 \\ Fax 703-605-6900 \\ E-mail info@ntis.gov \\ Website http://www.ntis.gov/help/ordermethods.aspx
}

Reports are available to DOE employees, DOE contractors, Energy Technology Data Exchange representatives, and International Nuclear Information System representatives from the following source:

Office of Scientific and Technical Information

PO Box 62

Oak Ridge, TN 37831

Telephone 865-576-8401

Fax 865-576-5728

E-mail reports@osti.gov

Website http://www.osti.gov/contact.html

This report was prepared as an account of work sponsored by an agency of the United States Government. Neither the United States Government nor any agency thereof, nor any of their employees, makes any warranty, express or implied, or assumes any legal liability or responsibility for the accuracy, completeness, or usefulness of any information, apparatus, product, or process disclosed, or represents that its use would not infringe privately owned rights. Reference herein to any specific commercial product, process, or service by trade name, trademark, manufacturer, or otherwise, does not necessarily constitute or imply its endorsement, recommendation, or favoring by the United States Government or any agency thereof. The views and opinions of authors expressed herein do not necessarily state or reflect those of the United States Government or any agency thereof. 
US DOE Fuel Cycle Research and Development Advanced Fuels Campaign

Report on fundamental modeling of irradiation-induced swelling and creep in FeCrAl alloys

\author{
Aaron Kohnert \\ Dwaipayan Dasgupta \\ Brian Wirth
}

Date Published: August 30, 2016

\author{
Prepared by \\ OAK RIDGE NATIONAL LABORATORY \\ Oak Ridge, TN 37831-6283 \\ managed by \\ UT-BATTELLE, LLC \\ for the \\ US DEPARTMENT OF ENERGY \\ under contract DE-AC05-00OR22725
}





\section{CONTENTS}

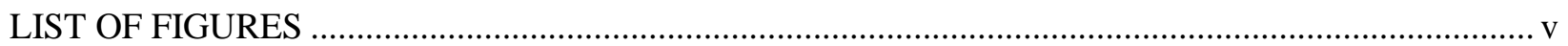

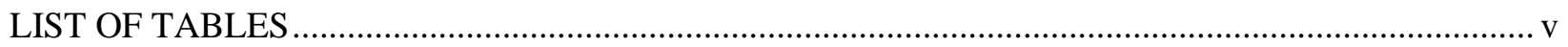

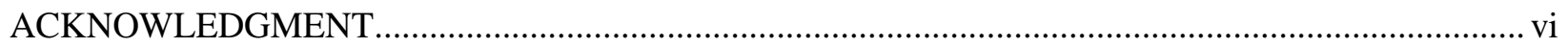

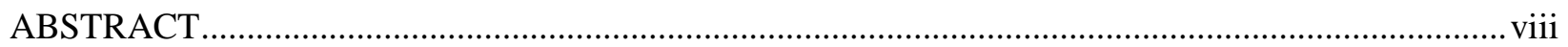

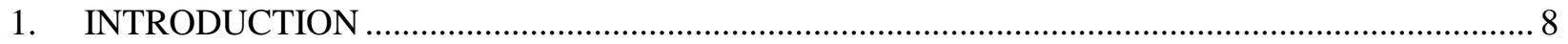

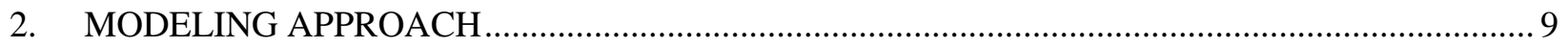

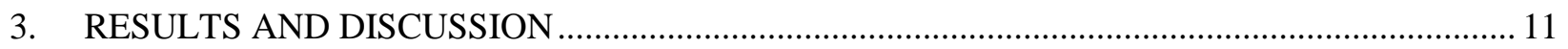

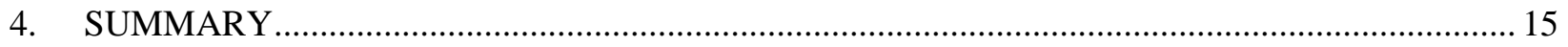

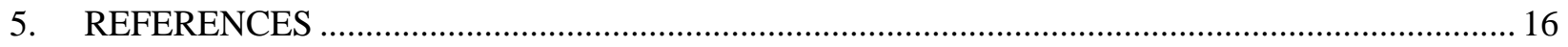





\section{LIST OF FIGURES}

Figure 1. Cluster dynamics model predictions of the net flux of interstitials to edge (left) or screw (right) dislocations for HFIR irradiation at a dose rate of $8.1 \times 10^{-7} \mathrm{dpa} / \mathrm{s}$ and a dose of 1.6 (blue) or 2.4 (red) dpa, as a function of irradiation temperature.

\section{LIST OF TABLES}

Table 1. Summary of experimental and modeling predictions of radiation damage microstructure in a $\mathrm{FeCrAl}$ alloy neutron irradiated to $1.8 \mathrm{dpa}$ at $382^{\circ} \mathrm{C}$ (experimental results obtained from Ref. [21]) and predicted by a cluster dynamics model at a dose of $1.62 \mathrm{dpa}$. 


\section{ACKNOWLEDGMENT}

The authors would like to thank Kevin Field and Kurt Terrani (Oak Ridge National Laboratory) for their collaboration and interaction with respect to experimental characterization of neutron irradiated $\mathrm{FeCrAl}$ specimens. The high-performance computing facilities of the Newton computing cluster at the University of Tennessee, are gratefully acknowledged. Research is sponsored by the Advanced Fuels Campaign of the Fuel Cycle R\&D program, Office of Nuclear Energy, US Department of Energy, under contract DEAC05-00OR22725 with UT-Battelle, LLC. 



\begin{abstract}
In order to improve the accident tolerance of light water reactor (LWR) fuel, alternative cladding materials have been proposed to replace zirconium ( $\mathrm{Zr}$ )-based alloys. Of these materials, there is a particular focus on iron-chromium-aluminum ( $\mathrm{FeCrAl}$ ) alloys due to much slower oxidation kinetics in high-temperature steam than $\mathrm{Zr}$-alloys. This should decrease the energy release due to oxidation and allow the cladding to remain integral longer in the presence of high temperature steam, making accident mitigation more likely. As a continuation of the development for these alloys, the material response must be demonstrated to provide suitable radiation stability, in order to ensure that there will not be significant dimensional changes (e.g., swelling), as well as quantifying the radiation hardening and radiation creep behavior. In this report, we describe the use of cluster dynamics modeling to evaluate the defect physics and damage accumulation behavior of $\mathrm{FeCrAl}$ alloys subjected to neutron irradiation, with a particular focus on irradiation-induced swelling and defect fluxes to dislocations that are required to model irradiation creep behavior.
\end{abstract}

\title{
1. INTRODUCTION
}

In the wake of events at the Fukushima Daiichi Nuclear Power Plant, interest has been revived in pursuing ironchrome-aluminum alloys as alternative nuclear fuel claddings to traditionally used zirconium-based alloys. This focus is largely driven by the search for better cladding performance during transient reactor operation where the fuel cladding is exposed to a high temperature steam environment.

During a severe accident scenario, such as a loss-of-coolant-accident, a nuclear reactor may lose its capacity to cool its fuel. The fuel rod temperatures begin to increases, quickly reaching temperatures where the coolant begins to evaporate. This lowers the coolant level in the core and eventually uncovers the fuel. Without sufficient heat transfer from the fuel rods to the coolant, the temperature of the fuel and cladding will increase dramatically. In the case of traditional zirconium-based alloys, as the temperature of the cladding reaches $\sim 1200^{\circ} \mathrm{C}$ the zirconium rapidly begins to oxidize with $\mathrm{H}_{2} \mathrm{O}$ in the coolant and steam. This oxidation reaction causes both thinning of the $\mathrm{Zr}$-alloy cladding as the metal reacts and releases large amounts of $\mathrm{H}_{2}$ gas into the reactor pressure vessel [1]. With prolonged exposure the cladding can weaken and subsequently rupture, releasing fission products into the coolant.

In order to increase the safety margin of LWR fuel in severe accident scenarios, several alternative cladding materials have been proposed to replace the currently used zirconium-based alloys. Of these materials, there is a particular focus on select iron-chrome-aluminum $(\mathrm{FeCrAl})$ alloys because they present slower oxidation kinetics in high-temperature steam than zirconium-based alloys [2]. This increased oxidation resistance may give more time to mitigate any further damage resulting from an accident.

Information is needed to determine the suitability of iron-chrome-aluminum alloys as fuel cladding and assess safety margins for their operation. In particular, these $\mathrm{Fe}-\mathrm{Cr}-\mathrm{Al}$ alloys will experience neutron irradiation and potential radiation effects on the dimensional stability and mechanical properties. In particular, the cladding material response must be demonstrated to provide suitable radiation stability, in order to ensure that there will not be significant dimensional changes (e.g., swelling), as well as quantifying the radiation hardening and radiation creep behavior. In this report, we describe the use of cluster dynamics to evaluate the defect physics and damage accumulation behavior of $\mathrm{FeCrAl}$ alloys, with a particular focus on irradiation-induced swelling and defect fluxes to dislocations that are required to model irradiation creep behavior.

This report fulfills fiscal year 2016 (FY16) milestone number M3FT-16OR020205033 within Work Package FT16OR02020503 at ORNL for the DOE-NE Advanced Fuels Campaign. 


\section{MODELING APPROACH}

Isolated vacancies and self-interstitial atoms (SIAs), as well as three-dimensional vacancy clusters and clusters of SIA in the form of planar, prismatic dislocation loops are continuously formed and will evolve in pure body-centered cubic iron under neutron irradiation [3-9]. A cluster dynamics (CD) model based on the reaction-diffusion rate theory is used here to predict the evolution of the vacancy and SIA defect clusters, which is a modified version of the model developed by $\mathrm{Xu}$ and Wirth [7, 10-12] and only considers the intrinsic defects and their clusters. Furthermore, since the probability that SIA and vacancy co-exist in a single cluster is very low due to their strong tendency for recombination, no mixed I-V clusters are considered, and it is thus sufficient to define any cluster using just an integer, with its absolute value being the number of point defects contained in the complexes and its sign ('-' for SIA clusters, or '+' for V-clusters) indicating the character of the cluster. Two numbers, NI and NV, are chosen as the number of interstitials in the largest SIA-cluster, and the number of vacancies in the largest Vcluster, respectively. Physically, these numbers prescribe the 'phase space' within which the clusters can interact with each other, and ensure the conservation of point defects. NI and NV should be chosen sufficiently large so that the computational results are not impacted by a prescribed phase space that is too small (e.g., insufficient cluster size to allow growth to large defect cluster size).

For neutron irradiation, it is common to model the system without a discrete spatial dependence, making use of the mean field approximation, as described here. However, for many cases of ion irradiation, and in particular for thinfilm ion irradiation studies, the spatial dependence of the damage profile, and the strong influence of the free surfaces, requires an explicit spatial dependence in the model, as fully described in References [7, 10-15]. As well, the nature of defect generation in metals induced by ions and neutrons is significantly different. The energy transfer cross section for ion - atom collisions is an atomic cross section $\left(\sim 10^{-17} \mathrm{~cm}^{2}\right)$ while that for neutrons is a nuclear cross section $\left(\sim 10^{-24} \mathrm{~cm}^{2}\right)$, and consequently, neutrons have a much larger range between collisions when travelling in a material. The damage production varies weakly along the depth direction for neutron irradiation and therefore, it is reasonable to treat the distribution of radiation damage production as homogeneous [16]. Thus, as noted previously, no explicit spatial dimension is necessary in this model, at least for low neutron dose levels before a clear spatial correlation of the defect microstructure develops.

Without an explicit spatial dependence, the concentration of each cluster is only a function of time, and ordinary differential equations describe the defect evolution. The binary reactions discussed in Refs. [7-12, 16] are still appropriate and become simpler, because there are only two types of defects (V-and SIA-clusters) considered during neutron irradiation. The generic form to describe the evolution of a cluster, without spatial dependence, is:

$$
\frac{d C_{i}}{d t}=\quad P_{i}+G_{-} T+G_{-} E \quad A_{-} T \quad A_{-} E,
$$

where $C_{i}$ refers to the volumetric concentration (in $1 / \mathrm{m}^{3}$ ) of the i-th cluster, is the neutron flux (in neutron $/ \mathrm{m}^{2} / \mathrm{sec}$ ), $P_{i}$ is the production 'probability' of the i-th cluster by neutron irradiation, $G$ refers to the collective generation rates, in which $G_{-} T$ refers to generation by trapping reactions and $G_{-} E$ refers to generation by emission, $A_{-} T$ indicates the annihilation of cluster $C_{i}$ by trapping reaction events, and $A \_E$ refers to annihilation by emission events. The detailed construction of the coupled system of ODEs is thus (for which $=[N I, N V]$ is the prescribed phase space, and for which we sum over clusters $m+p=i$ for forward reactions and $m$ reacts with $i$ in trapping reactions to reduce the concentration of species i): 


$$
\frac{d C_{i}}{d t}=P_{i}+k_{\substack{m+p=i \\ m, p 0 \\ m . p}} k_{m, p}^{+} C_{m} C_{P} \underset{\substack{m i \\ m i 0 \\ m . m+i}}{ } k_{m, i}^{+} C_{m} C_{i} \quad k_{i} C_{i}
$$

for $\mathrm{i}=\mathrm{NV}$ or $-\mathrm{NI}$, and

$$
\frac{d C_{i}}{d t}=P_{i}+k_{\substack{m+p=i \\ m, p \\ m, p}} k_{m, p}^{+} C_{m} C_{P}+k_{i+1} C_{i+1} \underset{\substack{m i \\ m 0 \\ m \cdot m+i}}{ } k_{m, i}^{+} C_{m} C_{i} \quad k_{i} C_{i}
$$

for $\frac{N V}{2}<i<N V$, and



for $2 i \frac{N V}{2}$, and

$$
\frac{d C_{i}}{d t}=P_{i}+\sum_{\substack{m+p=i \\ m, p 0 \\ m . p}} k_{m, p}^{+} C_{m} C_{P}+2 k_{2} C_{2}+\underset{\substack{m>2 \\ m}}{k_{m}} C_{m} \underbrace{}_{\substack{m i \\ m 0 \\ m \cdot m+i}} k_{m, i}^{+} C_{m} C_{i} \quad 2 k_{i, i}^{+}\left(C_{i}\right)^{2}
$$

for $i=1$, and

$$
\frac{d C_{i}}{d t}=P_{i}+\underset{\substack{m+p=i \\ m, p \\ m, p}}{ } k_{m, p}^{+} C_{m} C_{P}+2 k_{2} C_{2}+k_{\substack{m<2 \\ m}} k_{m} C_{\substack{m i \\ m<0 \\ m . m+i}} k_{m, i}^{+} C_{m} C_{i} \quad 2 k_{i, i}^{+}\left(C_{i}\right)^{2}
$$

for $i=1$, and

$$
\frac{d C_{i}}{d t}=P_{i}+\underset{\substack{m+p=i \\ m, p 0 \\ m . p}}{ } k_{m, p}^{+} C_{m} C_{P}+k_{i 1} C_{i 1} \underbrace{}_{\substack{m i \\ m i \\ m . m+i \\ m . m+i}} k_{m, i}^{+} C_{m} C_{i} \quad 2 k_{i, i}^{+}\left(C_{i}\right)^{2} \quad k_{i} C_{i}
$$

for $\frac{N I}{2} \quad i \quad 2$, and

$$
\frac{d C_{i}}{d t}=P_{i}+\underset{\substack{m+p=i \\ m, p \\ m, p}}{ } k_{m, p}^{+} C_{m} C_{P}+k_{i 1} C_{i 1} \underbrace{}_{\substack{m i \\ m 0 \\ m . m+i}} k_{m, i}^{+} C_{m} C_{i} \quad k_{i} C_{i}
$$


for $N I<i<\frac{N I}{2}$, where $k^{+}$is the forward reaction rate constant and $k$ is the backward reaction rate constant, having the same expressions as the classic rate theory derviations based on isotropic interactions and spherical reaction volumes as derived by Waite based on the original work of Smoluchowski, i.e.,

$$
\begin{gathered}
k_{m . p}^{+}=4 \quad\left(r_{m}+r_{p}\right)\left(D_{m}+D_{p}\right), \\
k=k^{+} C_{0} \exp \left(\frac{E_{b}}{k_{B} T}\right),
\end{gathered}
$$

where $r_{m}$ and $r_{p}$ are the trapping radii of clusters $m$ and $p, D$ is the diffusion coefficient of the reacting species, $C_{0}$ is the atomic number density of the iron matrix, $E_{b}$ is the binding energy of a single point defect to the cluster, $k_{B}$ is Boltzmann's constant, and $T$ is the temperature. Note that for emission $(C \rightarrow A+B)$, only those events in which at least one of the two products is a monomer (i.e., I or V) are considered since it is in general more energetically favorable for a cluster to emit a monomer than emit a dimer, trimer or a larger cluster, which is consistent with previous models [10-12].

The variations among equations (2)-(8) represent the computational details in the cluster dynamics modeling, which must be considered to ensure no computational artifacts (such as non-conservation of point defects) are introduced in the results. For example, the difference between Eq. (7) and (8) is that in Eq. (7) the interstitial clusters, $\mathrm{I}_{\mathrm{n}}$, where $\mathrm{n}$ is less than half of the pre-set maximum interstitial clusters size, are allowed to interact with similar sized clusters to generate a big cluster. However, in Eq. (7), this behavior is forbidden due to the fact that the resulting larger interstitial cluster would exceed the prescribed phase space. If we were able to solve an infinite number of equations/clusters in computation, this would not be necessary, but in reality, we can only solve finite, although fairly large, number of coupled equations and hence these detailed rules of computation have to be enforced.

\section{RESULTS AND DISCUSSION}

Here we present initial results of modeling the neutron irradiation behavior of an $\mathrm{Fe}-10 \mathrm{Cr}-5 \mathrm{Al}$ alloy, subject to neutron irradiation in the High Flux Isotope Reactor (HFIR) at an irradiation temperature of $\sim 380^{\circ} \mathrm{C}$ to a radiation dose of $1.8 \mathrm{dpa}$, with a dose rate of $8.1 \times 10^{-7} \mathrm{dpa} / \mathrm{s}$. We have assumed that the primary knock-on spectrum of the HFIR reactor in FeCrAl will produce displacement cascades with a similar population of vacancy and selfinterstitial clustering, consistent with displacement cascades produced in $\mathrm{Fe}$, e.g., no effect of $\mathrm{Cr}$ or $\mathrm{Al}$ additions on the initial cascade damage.

We have assumed an initial line dislocation density of $6.3 \times 10^{13} \mathrm{~m}^{-2}$, and that that population is either $100 \%$ edge dislocation component, or a mixture of $60 \%$ screw dislocation and $40 \%$ edge dislocation. The diffusion behavior of vacancy and self-interstitial atom clusters is consistent with our previous work on $\mathrm{Fe}$ and $\mathrm{Fe}-\mathrm{Cr}$ alloys $[16,19,20]$. We have also assumed that dislocation loops can form as a result of reactions that result in populations of either $\mathrm{a} / 2<111>$ or $\mathrm{a}<100>$ Burgers vectors, although the precise description of the mechanism and numerical treatment will be provided in a future milestone report. 
Table 1. Summary of experimental and modeling predictions of radiation damage microstructure in a $\mathrm{FeCrAl}$ alloy neutron irradiated to $1.8 \mathrm{dpa}$ at $382^{\circ} \mathrm{C}$ (experimental results obtained from Ref. [21]) and predicted by a cluster dynamics model at a dose of $1.62 \mathrm{dpa}$.

\begin{tabular}{|l|l|l|l|l|l|}
\hline & \multirow{2}{*}{$\begin{array}{c}\text { Experiment } \\
(1.8 \mathrm{dpa})\end{array}$} & \multicolumn{4}{|c|}{ Simulation (1.62 dpa) } \\
\cline { 3 - 6 } & & $\rho_{\mathrm{ui}}=0.63 \times 10^{14} \mathrm{~m}^{-2}$ & \multicolumn{2}{c|}{$\rho_{\mathrm{ui}}=2.5 \times 10^{14} \mathrm{~m}^{-2}$} \\
\hline Property & & Screw 0\% & Screw 60\% & Screw 0\% & Screw $60 \%$ \\
\hline $\mathrm{d}_{\mathrm{a}<100>}(\mathrm{nm})$ & $52.5 \pm 23.8$ & 41.1 & 89.9 & 51.2 & 77.4 \\
\hline$\rho_{\mathrm{a}<100>}\left(\mathrm{m}^{-3}\right)$ & $3.4 \pm 0.5 \times 10^{19}$ & $3.0 \times 10^{20}$ & $5.39 \times 10^{20}$ & $6.02 \times 10^{20}$ & $1.50 \times 10^{21}$ \\
\hline $\mathrm{d}_{\mathrm{a} / 2<111>}(\mathrm{nm})$ & $31.9 \pm 18.7$ & 3.14 & 2.9 & 2.72 & 2.62 \\
\hline$\rho_{\mathrm{a} / 2<111>}\left(\mathrm{m}^{-3}\right)$ & $2.6 \pm 0.6 \times 10^{20}$ & $3.15 \times 10^{20}$ & $3.38 \times 10^{19}$ & $8.46 \times 10^{18}$ & $6.94 \times 10^{16}$ \\
\hline$d_{\mathrm{bd}}(\mathrm{nm})$ & $9.1 \pm 3.2$ & 2.8 & 2.7 & 2.6 & 2.5 \\
\hline$\rho_{\mathrm{bd}}\left(\mathrm{m}^{-3}\right)$ & $1.0 \pm 0.1 \times 10^{20}$ & $1.02 \times 10^{22}$ & $9.06 \times 10^{21}$ & $8.03 \times 10^{21}$ & $5.52 \times 10^{21}$ \\
\hline
\end{tabular}

Table 1 shows the results obtained for the radiation damaged microstructure of the $\mathrm{FeCrAl}$ alloy following irradiation to a dose of $1.8 \mathrm{dpa}$ at a temperature of $382^{\circ} \mathrm{C}$, as determined by the transmission electron microscopy characterization of Fields and co-workers [21]. It is important to point out that experimentally, they were able to distinguish between large dislocation loops with Burgers vectors of both $a\langle 100\rangle$ and $a / 2\langle 111\rangle$, in addition to a fairly high number density of smaller, black dot features. Table 1 also includes the preliminary simulation results of our cluster dynamics model, for which we used two different initial line dislocation densities, namely $0.63 \times 10^{14}$ $\mathrm{m}^{-2}$ (experimental value), and a somewhat higher $2.5 \times 10^{14} \mathrm{~m}^{-2}$. As previously mentioned, we have also evaluated the effect of dislocation character of this pre-existing network, or line, dislocation sink for point defect absorption, considering the population either to be pure edge, or $60 \%$ screw and $40 \%$ edge dislocations. Notably, our model does predict the formation of quite large dislocation loops with Burgers vector of $a\langle 100\rangle$, although the number densities are generally more than an order of magnitude larger than experimentally observed. However, our model is currently incapable of predicting the growth of very large dislocation loops with a Burgers vector of a/2<111>. This results from the trap mediated diffusion behavior that we have assumed for larger, prismatic type selfinterstitial clusters, and indicates the possibility that some microstructural feature may be responsible for trapping such loops. Interestingly, the number density of the $a / 2<111>$ loops that our cluster dynamics model predicts is within the expected experimental range. We also predict a very high density of black dot features, which is not consistent with the experimental observations.

Notably, as we increase the pre-existing dislocation density in the model, the predicted observable dislocation loop densities do decrease, and our current hypothesis is that we do not include a defect sink in the model that is responsible for increasing the amount of point defect recombination events, and thereby limiting the direct accumulation of self-interstitial type defects in prismatic dislocation loops. We continue to evaluate the possibilities for this, including that other precipitates formed in the microstructure, such as Cr-rich alpha prime may serve as these defect sink recombination centers. 

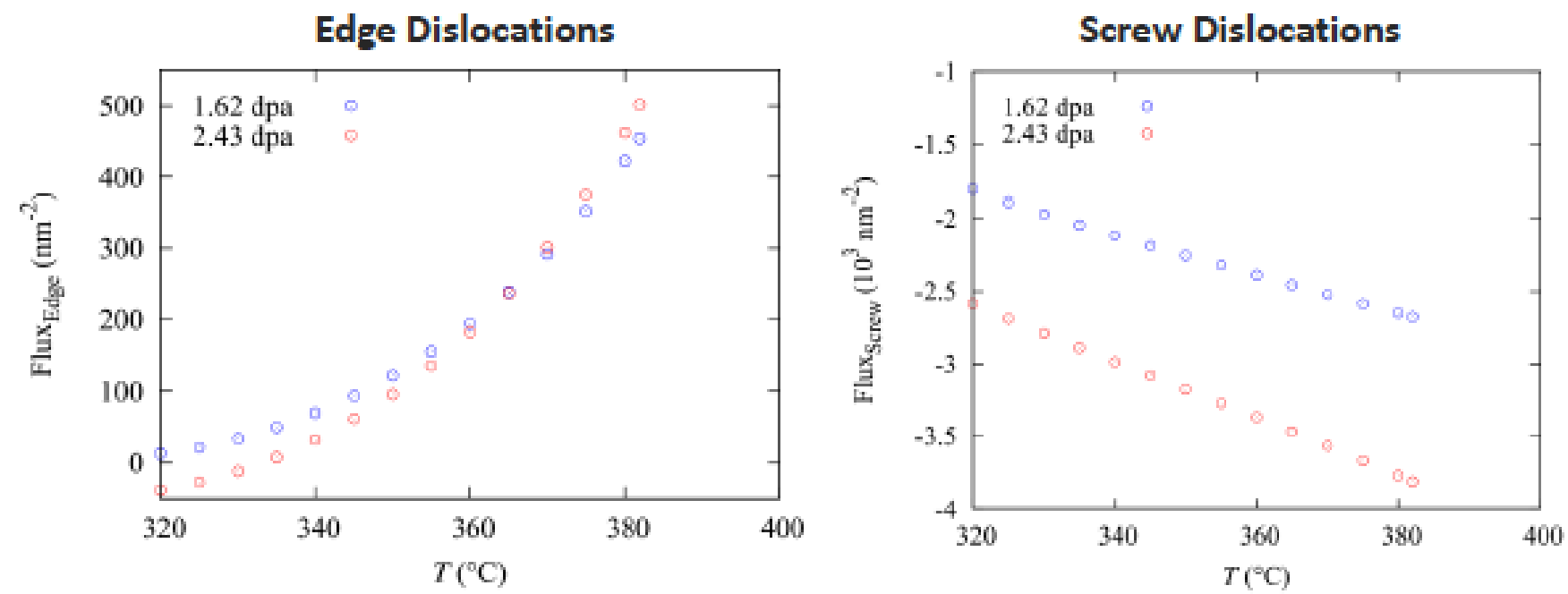

Figure 1. Cluster dynamics model predictions of the net flux of interstitials to edge (left) or screw (right) dislocations for HFIR irradiation at a dose rate of $8.1 \times 10^{-7} \mathrm{dpa} / \mathrm{s}$ and a dose of 1.6 (blue) or 2.4 (red) dpa, as a function of irradiation temperature.

Just as notable, in the cluster dynamics simulations that we have performed thus far, there is no indication of cavity nucleation that could drive void swelling or other dimensional instability. However, much more modeling work and analysis is required to confirm this preliminary conclusion that void swelling will not be a problem for the FeCrAl based fuel cladding for LWR applications.

One other attribute of this modeling effort that we have focused on relates to calculating the net point defect fluxes to the edge versus screw dislocations, so that we can quantify the amount of dislocation climb driven by the radiation damage process. Figure 1 shows the initial model predictions for the net flux of point defects to edge (left) versus screw (right) dislocations during HFIR irradiation at $8.1 \times 10^{-7} \mathrm{dpa} / \mathrm{s}$ to a dose of about 1.6 or $2.4 \mathrm{dpa}$, as a function of temperature. The model predictions indicate a strong partitioning of self-interstitial atom defects to the edge dislocation, whereas a very strong flux of vacancy defects is absorbed at the screw dislocation (+ units refer to net self-interstitial absorption, whereas negative values refer to vacancy absorption). Clearly, a strong temperature dependence is also predicted by the model, with a significant increase in the self-interstitial flux with increasing temperature.

\section{SUMMARY}

The work presented herein focused on describing a cluster dynamics modeling approach to assess the radiation stability of $\mathrm{FeCrAl}$ based fuel clad, under consideration as an accident tolerant fuel. The preliminary model results indicate the ability of the model to predict the evolution of dislocation loops with both $\mathrm{a} / 2<111\rangle$ and $\mathrm{a}<100\rangle$ Burgers vectors, although the preliminary results are not entirely in agreement with available experimental characterization. Notably, however, the models indicate that there should not be any concern for void swelling or dimensional instability of the FeCrAl. Our cluster dynamics models can also calculate the net flux of point defects to fixed dislocation sinks in the material, which offer the possibility of connecting to models of irradiation creep of $\mathrm{FeCrAl}$ alloys, assuming a climb mediated dislocation glide interaction mechanism dominates the irradiation creep mechanism in this temperature and stress regime. Future efforts will continue to refine the model, as well as the assumed defect production in displacement cascades, and the thermodynamics and kinetics of defect properties in FeCrAl alloys, with the promise of predicting the radiation response of these alloys in prototypic LWR environments. 


\section{REFERENCES}

[1] P. Hofman: Current knowledge of core degradation phenomena. Journal of Nuclear Materials 270 (1998) 175-181.

[2] K.A. Terrani, S.J. Zinkle, and L.L. Snead: Advanced oxidation-resistant iron-based alloys for LWR fuel cladding. Journal of Nuclear Materials 448 (2014) 420-435.

[3] G.J. Ackland, D.J. Bacon, A.F. Calder, and T. Harry: Computer simulation of point defect properties in dilute Fe-Cu alloy using a many-body interatomic potential. Phil. Mag. A 75, 713 (1997).

[4] R.E. Stoller, G.R. Odette, and B.D. Wirth: Primary damage formation in bcc iron. J. Nucl. Mater. 251, 49 (1997).

[5] W.J. Phythian, R.E. Stoller, A.J.E. Foreman, A.F. Calder, and D.J. Bacon: A comparison of displacement cascades in copper and iron by molecular dynamics and its application to microstructural evolution. J. Nucl. Mater. 223, 245 (1995).

[6] N. Soneda and T. Diaz de la Rubia: Defect production, annealing kinetics and damage evolution in $\alpha-$ Fe: An atomic-scale computer simulation. Phil. Mag. A 78, 995 (1998).

[7] D.H. Xu, B.D. Wirth, M.M. Li, and M.A. Kirk: Combining in situ transmission electron microscopy irradiation experiments with cluster dynamics modeling to study nanoscale defect agglomeration in structural metals. Acta Mater. 60, 4286 (2012)

[8] C.J. Ortiz and M.J. Caturla: Simulation of defect evolution in irradiated materials: Role of intracascade clustering and correlated recombination. Phys. Rev. B 75, 184101 (2007).

[9] C.J. Ortiz, P. Pichler, T. Fuhner, F. Cristiano, B. Colombeau, N.E.B. Cowern, and A. Claverie: A physically based model for the spatial and temporal evolution of self-interstitial agglomerates in ion implanted silicon. J. Appl. Phys. 96, 4866 (2004).

[10] D. Xu and B.D. Wirth: Spatially dependent rate theory modeling of thermal desorption spectrometry of helium-implanted iron. Fusion Sci. Technol. 56, 1064 (2009).

[11] D. Xu and B.D. Wirth: Modeling spatially dependent kinetics of helium desorption in BCC iron following He ion implantation. J. Nucl. Mater. 403, 184 (2010).

[12] D. Xu, X. Hu, and B.D. Wirth: A phase-cut method for multi-species kinetics: Sample application to nanoscale defect cluster evolution in alpha iron following helium ion implantation. Appl. Phys. Lett. 102, 011904 (2013).

[13] D. Xu, B.D. Wirth, M. Li, and M.A. Kirk: Defect microstructural evolution in ion irradiated metallic nanofoils: Kinetic Monte Carlo simulation versus cluster dynamics modeling and in situ transmission electron microscopy experiments. Appl. Phys. Lett. 101, 101905 (2012).

[14] D. Xu, B.D. Wirth, M. Li, and M.A. Kirk: Recent work towards understanding defect evolution in thin molybdenum foils through in situ ion irradiation under TEM and coordinated cluster dynamics modeling. Current Opinion in Solid State Materials Science 16, 109 (2012).

[15] M. Li, M.A. Kirk, P.M. Baldo, D. Xu, and B.D. Wirth: Study of defect evolution by TEM with in situ ion irradiation and coordinated modeling. Phil. Mag. 92(16), 2048 (2012).

[16] X. Hu, D. Xu, T.S. Byun, and B.D. Wirth: Modeling of irradiation hardening of iron after low-dose and low-temperature neutron irradiation. Modeling and Simulation in Materials Science \& Engineering 22, 0655002 (2014).

[17] M.V. Smoluchowski: A mathematical theory of coagulation kinetics of colloidal solutions. Z. Physik Chem 92, 192 (1917).

[18] T.R. Waite: Theoretical treatment of the kinetics of diffusion-limited reactions. Phys. Rev. B 107, 463 (1957).

[19] A.A. Kohnert and B.D. Wirth: Cluster dynamics models of irradiation damage accumulation in ferritic iron. I. Trap mediated cluster diffusion. Journal of Applied Physics 117 (2015) 154305. 
[20] A.A. Kohnert and B.D. Wirth: Cluster dynamics models of irradiation damage accumulation in ferritic iron. II. Effects of reaction dimensionality. Journal of Applied Physics 117 (2015) 154306.

[21] K.G. Field, X. Hu, K.C. Littrell, Y. Yamamoto, L.L. Snead: Radiation tolerance of neutronirradiated model Fe-Cr-Al Alloys. Journal of Nuclear Materials 465 (2015) 746-755. 